\title{
Experiencias de la relación universidad-ciencia-desarrollo en los estudios sociales rurales en Cuba
}

Yisel Herrera-Martínez y Alicia Martínez-León

\section{RESUMEN}

Es frecuente, en los últimos años, el estudio sobre la tercera misión de la educación superior y la pertinencia social de las universidades; varios son los modelos teóricos y las contribuciones empíricas. La comunicación analiza la interrelación entre el desarrollo científico en un campo de producción y legitimación de saberes particulares: los estudios sociales rurales y la educación superior. El uso de la sociología del conocimiento como instrumento teórico-metodológico devela las implicaciones y determinaciones sociales de la producción de conocimientos, dimensiones fundamentales en esta relación. Así, las ciencias sociales ofrecen una perspectiva diferente en el cumplimiento de la tercera misión.

Palabras clave: pertinencia social, desarrollo científico, relación universidad-sociedad, ciencias sociales, estudios sociales rurales, Cuba.

\section{Yisel Herrera Martínez}

Cubana. Doctora en Ciencias Sociológicas por la Universidad de La Habana, Cuba. Profesora auxiliar del Departamento de Estudios Socioculturales de la Universidad de Cienfuegos "Carlos Rafael Rodríguez". Temas de investigación: intervención social comunitaria en espacios rurales, sociología del conocimiento científico, estudios sociales de la ciencia y la tecnología. ORCID: https://orcid. org/0000-0003-2205-7684. 


\section{Experiencias da relação universidade-ciência-desenvolvimento nos estudos sociais rurais em Cuba}

\section{RESUMO}

É frequente, nos últimos anos, o estudo sobre a terceira missão da educação superior e a pertinência social das universidades; vários são os modelos teóricos e as contribuições empíricas. A comunicação analisa a inter-relação entre o desenvolvimento científico em um campo de produção e legitimação de saberes particulares: os estudos sociais rurais e a educação superior. O uso da sociologia do conhecimento como instrumento teórico-metodológico mostra as implicações e determinações sociais da produção de conhecimentos, dimensões fundamentais nesta relação. Asim, as ciências sociais oferecem uma perspectiva diferente na realização da terceira missão.

Palavras chave: pertinência social, desenvolvimento científico, relação universidade-sociedade, ciências sociais, estudos sociais rurais, Cuba.

\section{Experiences of the university-science-development relationship in rural social studies in Cuba}

\section{ABSTRACT}

In recent years, many studies have been carried out about the third mission of higher education and the social relevance of universities, based on several theoretical models and empirical contributions. This paper analyzes the interrelation between scientific development in a particular field of production and the legitimization of knowledge, between the rural social studies and higher education. The use of sociology of knowledge as a theoretical-methodological instrument reveals the social implications and determinations of knowledge production, that are key dimensions in this relationship. In that way, the social sciences offer a different perspective in the fulfillment of the third mission.

Key words: social relevance, scientific development, university-society relationship, social sciences, rural social studies, Cuba. 


\section{Introducción}

Muchos han sido los estudios y valoraciones, en las últimas décadas, acerca del papel de la educación superior y de las universidades en el desarrollo de la sociedad. Sin importar ideologías, existe un consenso reconocido a partir del Congreso Mundial de Educación Superior de París de 1998 (UNESCO, 1998) sobre el papel de este nivel de enseñanza como un eslabón esencial para el desarrollo social y para el crecimiento económico. A estos valores se les suman otros de carácter más emancipador: el fortalecimiento de la identidad cultural para mantener la coherencia social, la lucha contra la pobreza y por el desarrollo de una cultura de paz.

Para la consecución de los valores, la educación superior precisa de una evaluación constante de sus instituciones y de su organización social en función de la adaptación a los cambios y demandas que operan en la sociedad. Por tanto, se introduce la perspectiva de la pertinencia social y la calidad de los procesos sustantivos y surge un requerimiento fundamental: el papel de la educación superior y su contribución al desarrollo.

En el contexto latinoamericano el problema del desarrollo ocupó un lugar primordial en las agendas políticas y científicas durante las décadas del cincuenta al setenta del siglo XX. El estructuralismo, respaldado por los trabajos realizados en el marco de la Comisión Económica para América Latina (CEPAL) y las teorizaciones sobre la dependencia, determinó problemas socioeconómicos y sociopolíticos, además de la interrelación con procesos culturales, que conllevaban a un desarrollo inferior de la ciencia, la tecnología y la innovación (Núñez, 1999). En todo proceso de intervención: políticas públicas, formación de profesionales y fuerza calificada, era necesario articular la investigación científica.

Lo anterior da lugar al surgimiento de un pensamiento latinoamericano sobre ciencia, tecnología y desarrollo, emergente en los sesenta y setenta, representado, entre otros, por Jorge Sábato, Amílcar
Herrera, César Varsavsky, Miguel Wionseck, Máximo Halty, Francisco Sagasti, Osvaldo Sunkel, Marcel Roche, José Leite Lopes y que sirve de antecedente al nuevo enfoque presentado como respuesta a la agudización de las desigualdades generadas bajo la implementación de políticas neoliberales en América Latina en los noventas, la "transformación productiva con equidad" (CEPAL, 1990). Este pensamiento concede especial importancia a la educación y al conocimiento, considerado este último como elemento central del nuevo paradigma productivo, por lo que la transformación educativa es esencial (CEPALUNESCO, 1992).

Esta trayectoria antecede a la problemática fundamental que origina la reflexión presentada en este artículo: la relación entre educación superior y el conocimiento científico a partir de una experiencia particular en Cuba. La riqueza de los estudios rurales y agrarios en las ciencias sociales cubanas muestra la permanencia de estos temas en el tiempo y en la agenda científica; descubre su centralidad en el devenir histórico, económico, socio-político y cultural del país.

La universidad cubana, luego de 1962, con la Reforma Universitaria, asume un compromiso social con el desarrollo científico y económico-social de la nación y rompe con el modelo napoleónico, tradicional, de la formación de profesionales, al asumir la tercera misión de las universidades como un recurso fundamental para el desarrollo de la nación. La integración de la docencia, la investigación y la extensión universitaria posibilita la articulación de actores, la utilización de la ciencia y la innovación para lograr la transformación social.

Aunque la política económica implementada por el Estado revolucionario a partir de 1959 se ha encaminado a diversificar la producción agrícola y utilizar de forma eficiente las ventajas que ofrece la agricultura para sustituir importaciones y crear una base alimentaria nacional, las investigaciones realizadas principalmente en las universidades del país 
presentan dificultades para situarse como significativas en las transformaciones económico- sociales que demanda el contexto.

Con la elaboración de la Política Científica Nacional se promueve la aplicación de los resultados investigativos, su introducción en la práctica y aboga por la integración producción-docencia-investigación (Partido Comunista de Cuba, I Congreso 1978: 138). La pertinencia de la extensión universitaria en vínculo directo con sus misiones tradicionales puede convertir los resultados de las ciencias sociales en instrumentos de diagnóstico, acompañamiento y/o pronóstico de políticas públicas para el desarrollo. Pero sólo conociendo las determinaciones e implicaciones sociales de ese conocimiento producido es posible dar cuenta de las transformaciones y sus complejidades $^{1}$ y debe influir en el establecimiento de una cooperación coherente entre ciencia y sociedad.

La sociología del conocimiento, como estrategia teórico-metodológica, propone que la reflexividad del conocimiento producido y la legitimación del mismo, es fundamental en el análisis de las determinaciones e implicaciones sociales del saber científico. Así las ciencias sociales contribuyen desde una perspectiva particular al análisis del desarrollo científico y la educación superior.

En los estudios sociales sobre el medio rural se constata la presencia de una dispersión disciplinar, institucional y geográfica, y se identifican diferentes tipos de discursos producidos por las universidades, los centros de investigación adscritos al Sistema de Ciencia Tecnología e Innovación (SCTI) y por los organismos de la administración central del Estado (OACE). Aquí radica la contradicción científica que permite establecer como objetivo el análisis de las determinaciones sociales e implicaciones de la producción de conocimientos sobre lo rural, por las ciencias sociales cubanas en la relación entre el desarrollo científico y la educación superior.

$\mathrm{El}$ artículo se estructura en un acápite que aborda los elementos teóricos que permiten comprender el papel de las universidades en el desarrollo de un país. La pertinencia social, las misiones de la universidad, y modelos y teorías de vinculación de actores son referentes principales. El epígrafe de materiales y métodos ofrece a la sociología del conocimiento como un elemento de la reflexividad que sustenta la perspectiva de análisis. Se presentan además los resultados de las investigaciones sociales acerca de lo rural en Cuba potenciando las determinaciones e implicaciones sociales del conocimiento.

\section{Elementos teóricos: presupuestos para entender la relación educación superior, ciencia y desarrollo}

Las instituciones de educación superior, las universidades, poseen funciones que permiten la interrelación continua con todos los ámbitos de la vida social. Pero es la Reforma de Córdoba, Argentina, en 1918, la que marca un punto de giro en las funciones tradicionales de formación: poner el saber universitario al servicio de la sociedad y convertir sus problemas en tema prioritario de sus preocupaciones a través de la extensión universitaria vinculada con la investigación.

En la Declaración de la Unesco (1998), realizada en el marco de la Conferencia Mundial sobre Educación Superior, se afirma que la pertinencia de la educación superior debe evaluarse en términos de la adecuación entre lo que la sociedad espera de las instituciones y lo que éstas hacen. Así, para la extensión universitaria o la tercera misión de las universidades, se legitiman procesos que ya eran práctica social y

\footnotetext{
${ }^{1}$ Los objetivos fundamentales en la política científica en el campo de las ciencias sociales se enmarcan en los trabajos encaminados a lograr la elevación del carácter científico de la dirección de la sociedad y su consecuente desarrollo a partir de las leyes generales de la construcción del socialismo y el comunismo (Partido Comunista de Cuba, 1978) y puesta en vigor de la Resolución $119 / 1985$ de la Academia de Ciencias de Cuba (ACC).
} 
que tienen particularidades en el contexto latinoamericano; se inicia una tradición que apuesta por el cambio educativo y cultural a partir de una transformación social y política de la sociedad.

Sin embargo, esta perspectiva coexiste con otros modelos (Serna, 2007): el modelo altruista concibe la extensión como acciones desinteresadas de los universitarios en favor de las poblaciones marginadas. $\mathrm{El}$ divulgativo intenta definir procedimientos por medio de los cuales se pueden acercar a la población los adelantos técnicos y las expresiones culturales producidas por la universidad; sitúa a la universidad como centro de poder que no tiene presentes las necesidades del contexto. El modelo concientizador apela a la creación de conciencia, al despertar de la capacidad de análisis crítico y la acción transformadora, lo que desemboca en una participación activa. El vinculatorio empresarial cree que la labor de la universidad debe estar enfocada a satisfacer las necesidades de las empresas; como consecuencia tiene una tendencia mercantilista y la pérdida del sentido social con el que fue planteada la función de extensión.

En Cuba, con la Reforma universitaria (1962), inicia la integración de la universidad con el pueblo y se legitima, con la práctica social, la función totalizadora planteada por González y González (2003). Ésta se manifiesta en cada uno de los procesos y eslabones estructurales de la educación, perfeccionando los mecanismos de interacción y transformación del espacio en que se inserta. El Programa de desarrollo de la Extensión Universitaria creado en 1988, consolida las interacciones de las universidades al interior y fuera de sus espacios físicos. La transformación social y la solución de necesidades se convierten en el objetivo de una relación bidireccional entre universidad y sociedad.

Los proyectos extensionistas dirigidos al desarrollo sociocultural comunitario son el eje articulador de un modelo de desarrollo integral, de acuerdo con la clasificación de González y González (2006), que proyecta a la universidad como una institución crítica y creativa, que parte del concepto de la democratización del saber. Este modelo integral presente en Cuba, es superior al tradicional que coincide con el primero de Serna (2007), y al economiscista, semejante al vinculatorio empresarial.

El proceso analítico que sigue el presente texto, la articulación de funciones universitarias y sus procedimientos, debe combinarse con los diferentes modelos, enfoques y teorías relacionados con los componentes de los sistemas de ciencia e innovación, los actores y redes que permiten una vinculación entre el conocimiento producido, legitimado y difundido, a partir de la relación ciencia-educación superior-desarrollo.

La periodización planteada por Etzkowitz (2004) alude a una primera revolución académica, con la incorporación de la investigación en las universidades. Este giro hacia la investigación se profundiza debido a una mayor intervención de la universidad en los procesos económicos y sociales y a los vínculos con el sector empresarial con impactos en la capitalización del conocimiento (Etzkowitz y Leydesdorff, 1997). Se pasa así de un modelo lineal centrado más en la calidad de la oferta de conocimientos por las universidades a un modelo que potencia las demandas sociales que eventualmente el conocimiento debe satisfacer y que ha sido denominado como contexto-céntrico.

Este modelo es un modo emergente que contempla la generación del conocimiento en el contexto de su aplicación e implicaciones: es el resultado de un esfuerzo transdisciplinario, de la inclusión y participación de la diversidad de actores y organizaciones del contexto, de la apropiación social en el proceso de su generación que se deriva de su reflexividad y compromiso social (Souza et al., 2001).

Las interrelaciones entre algunos de estos actores se exponen en las formulaciones de Sábato y Botana (1975) y en el modelo de la Triple Hélice; ellos exponen que la superación del subdesarrollo de América Latina resultará de la acción simultánea de diferentes 
políticas y estrategias, asimismo la investigación científico-tecnológica es una poderosa herramienta de transformación de una sociedad. Estos presupuestos son la base para establecer una relación fuerte y permanente entre diseñadores y ejecutores de la política, quienes ofertan la investigación científica y los demandantes de tecnología.

\begin{abstract}
Enfocada como un proceso político consciente, la acción de insertar la ciencia y la tecnología en la trama misma del desarrollo significa saber dónde y cómo innovar. La experiencia histórica demuestra que este proceso político constituye el resultado de la acción múltiple y coordinada de tres elementos fundamentales en el desarrollo de las sociedades contemporáneas: el gobierno, la estructura productiva y la infraestructura científico-tecnológica (Sábato y Botana, 1975: 5).
\end{abstract}

Este modelo, implícitamente, reconoce al sistema de educación superior, pero como un componente de la infraestructura científico-tecnológica, lo limita a formar en calidad y cantidad a los hombres que protagonizan la investigación. El rol investigativo de los centros de educación superior y la articulación de este proceso con la formación de calidad queda relegado a un servicio, no a una misión que contribuye al desarrollo.

Con la Triple Hélice, los centros de educación superior adquieren relevancia al interconectarse con la industria y el gobierno. Este modelo es ampliamente reconocido por su importancia como base institucional dinámica para la creación de riqueza (en el sentido de bienestar) y el desarrollo sustentable a través de la generación de conocimiento y su efectiva aplicación. Cuenta con tres etapas: en la etapa I, el Estado abarca la industria y la academia, además regula las relaciones entre las esferas institucionales; en la etapa II, existe una separación de las esferas institucionales y se circunscriben las relaciones entre ellas; la etapa III refleja la generación de una infraestructura de conocimiento en función de la interrelación de las esferas institucionales (Etzkowitz y Leydesdorff, 2000).

Este modelo contribuye a fundamentar la tercera misión de las universidades, la extesnsión, pues además de la docencia y la investigación, su contribución al desarrollo social y económico, implementa un variado repertorio de mecanismos de vinculación con las empresas (Casas, 2015). Con la tercera misión, las instituciones de educación superior deben trabajar sobre la transferencia de conocimiento incorporado en estudiantes de doctorado y graduados - transferencia de competencias entrenadas a través de la investigación a la industria y servicios públicos orientados por misiones y también por el emprendedurismo (spin-offs); la propiedad intelectual; los contratos con la industria a través de la coproducción de conocimiento y su circulación a la industria; los contratos con cuerpos públicos para satisfacer la dimensión de servicio público de las actividades de investigación; la participación en la elaboración y/o implementación de políticas a diferentes niveles y sobre todo, el involucramiento de la universidad en la vida social y cultural (Schoen et al., 2006).

Sobre la base de estas propuestas se amplían los indicadores de pertinencia e importancia social y los fundamentos en los que descansa la gestión universitaria. Sin embargo, para un análisis de la interrelación objeto de análisis es necesario significar los modelos conceptuales de producción de conocimiento: el modo 1 y modo 2 (Batista y Pérez, 2016). Como consecuencia, los indicadores para evaluar la productividad, legitimidad y difusión del conocimiento cambian.

En el modo 1 el conocimiento es generado dentro del contexto disciplinar cognitivo, para solucionar problemas en un contexto gobernado por intereses académicos. Se caracteriza por la homogeneidad de prácticas científicas que respetan la organización jerárquica. En el modo 2 el conocimiento es generado en contextos transdisciplinares sociales y económicos más amplios, cuestiona el papel de las instituciones 
dedicadas a producir conocimiento (universidades, instituciones gubernamentales de investigación). Se produce bajo la negociación de intereses de diversos actores y demandas sociales; la heterogeneidad conduce a prácticas sociocognitivas que suponen la integración de las habilidades consensuadas y la difusión y la socialización de los resultados trascienden los canales institucionales (Gibbons et al., 1997).

Los modos de producción de conocimiento en el contexto cubano son variados y plantean formas diferenciadas de concebir la autoridad científica; las relaciones de poder frente a actores determinantes en la toma de decisiones condicionan la autonomía en términos institucionales u organizacionales. Los procesos cognitivos realizados por las universidades en actividades de docencia, investigación y extensión generan estos procesos organizacionales que reproducen la naturaleza contradictoria de los procesos económicos y científico-tecnológicos de un país subdesarrollado: insuficientes espacios interactivos de aprendizaje, transformación social y de articulación actores; la productividad y difusión del conocimiento científico es frecuente sólo en los circuitos académicos y científicos.

En un balance sobre los referentes teóricos expuestos, los autores consultados aseveran que el modo 1 se ha convertido en el modo de producción característico de la investigación institucionalizada en las universidades profesionales. ${ }^{2}$ Plantean además que estos modelos son generales, conceptuales y describen los procesos de producción de conocimientos, pero no explicitan un sistema de conocimientos estructurados y sistematizados que analice y explique teóricamente el sistema universitario, y permita una mayor flexibilidad teórica, metodológica y práctica de la gestión (Batista y Pérez, 2016).

Sobre esta base parten los resultados que ofrece esta comunicación, potencia el análisis desde los nexos internos establecidos en la producción y legitimación del conocimiento resultado de la investigación científica que asumen los colectivos universitarios para hacerse corresponder con la pertinencia social de la universidad. Asimismo, escudriña sobre la interdependencia entre los procesos de formación, investigación y extensión como determinantes de la calidad de los procesos universitarios y el desarrollo de la sociedad; éstos no pueden identificarse como una relación de causa-efecto.

Se debe tener presente las problemáticas planteadas en el Informe Mundial de la Organización de las Naciones Unidas para la Educación, la Ciencia y la Cultura (UNESCO, 2005): concentración de recursos en las universidades o departamentos con mejores resultados o incluso separación de las funciones de investigación y enseñanza, en nombre del principio de la rentabilidad de la inversión; promoción de las disciplinas más avanzadas, especialmente en el sector clave de la ciencia y tecnología (nuevas tecnologías de la información y la comunicación, biotecnologías y nanotecnologías), en detrimento de las humanidades; fomento de modalidades de gestión más empresariales.

Según Coraggio (2002), la articulación de la educación superior con el conocimiento científico originado por la investigación y el desarrollo, produce demandas y nuevos retos. La conjunción del conocimiento científico de los especialistas y de los saberes prácticos de los actores sociales, se encuentra ya no en un puro diálogo de reconocimiento mutuo sino en un espacio de decisión democrática, de planificación estratégica y gestión pública participativa. Esto supone reducir la incomunicación entre los expertos y los ciudadanos, cambiar las disposiciones a dialogar, desechando el tecnocratismo y facilitar que los sectores no organizados ejerzan una participación activa.

\footnotetext{
${ }^{2}$ Saborido (2018) plantea que en la enseñanza superior coexisten diferentes tipos de universidades que corresponden al modelo napoleónico francés: las universidades profesionales, y al paradigma de los hermanos Humboldt: las universidades de investigación y universidades científicas y tecnológicas.
} 
Las universidades, al dirigir sus agendas hacia el desarrollo, deben investigar y reflexionar críticamente acerca de sus estrategias de formación, sus métodos de investigación y su responsabilidad para que el conocimiento que producen sea apropiado por y para beneficio de toda la sociedad. En este orden, el fortalecimiento del modelo de extensión agraria ${ }^{3}$ debe adaptarse a la diversificación de las demandas; generar, y utilizar nuevos enfoques, paradigmas y conocimientos en función de la multifuncionalidad del espacio rural. Así, el saber acumulado en el pre y posgrado en las universidades y por el Instituto Nacional de Ciencias Agrícolas, coadyuva a solucionar problemas productivos y de la vida cotidiana.

La extensión universitaria en estos espacios, sobre todo con el Proyecto de innovación agropecuaria local (PIAL), ${ }^{4}$ comparte la concepción del campo de estudios sociales rurales que no limita el desarrollo agrario, sino que visualiza las complejas relaciones sociales y culturales que se establecen en relación con otras actividades rurales no agropecuarias, la pluriactividad, la vida cotidiana. Así lo rural, categoría socio-espacial (Entrena, 1998), sostiene un espacio físico con diversas formas de ocupación del territorio, de dominación social y de uso de recursos naturales que discurren en actividades económicas con base principal, pero no exclusiva, en el sector primario de la economía en continuum con lo urbano. También se convierte en espacio de vida, donde relaciones y estructuras sociales, económicas, políticas y culturales se construyen en relación recíproca con el medio natural.

\section{Materiales y métodos}

El análisis que se presenta constituye una reconstrucción analítica sobre un estudio precedente que adopta a la sociología del conocimiento como perspectiva teórico- metodológica. Ésta repiensa el conocimiento a partir de la sociedad que lo origina y del devenir de las ideas resultantes de este proceso; produce un conocimiento desde el propio conocimiento y devela los procesos de apropiación que los actores sociales involucrados realizan en los diferentes espacios sociales.

El saber sociológico adquiere utilidad y valor académico y extraacadémico. La comunidad científica y los actores sociales definen la importancia y la utilización de este conocimiento a partir de la reproducción del conocimiento legitimado. Se utilizan los saberes para generar otros saberes, las contribuciones de las ciencias sociales pueden generalizarse hacia la investigación aplicada y experimental y sobre todo, a la transformación de contextos culturales y socioeconómicos rurales. En lo académico ocurren transformaciones epistemológicas, se desarrollan la creatividad, la innovación, el trabajo en equipo, la competencia, la planificación y evaluación de una investigación que se exige transdiciplinaria.

Como artículo de reflexión basa su análisis en los datos primarios que provienen de una base de datos que recoge 278 producciones científicas acerca de lo rural en Cuba, estas producciones científicas se ajustan a la acepción restringida de ciencias sociales (Martín, 1999): el enfoque sociológico, ${ }^{5}$ antropología sociocultural, lingüística, demografía. También se

\footnotetext{
${ }^{3}$ La extensión agraria, es un instrumento para fortalecer la capacidad de autoaprendizaje e innovación de las comunidades rurales hacia la competitividad y la sostenibilidad, es un proceso integral que va más allá que el apoyo técnico (Engel, 2001). En Cuba coexisten diferentes formas de extensión (Pavón, 2015) y las experiencias son desarrolladas principalmente por profesionales de las ciencias agropecuarias y el Sistema de Extensión Agraria en Cuba, y responden a diversos programas y proyectos.

${ }^{4}$ Co-innovación... Véanse publicaciones como Impactos en Cuba del programa de innovación agropecuaria: aprendizaje a ciclo completo", compilación, Cuba, Editorial Feijóo, 2011; Innovación social y desarrollo local: documentación y sistematización de experiencias, compilación, Cuba, Ediciones Luminaria, 2013; Jaime García et al. (2016), Sector cooperativo y desarrollo local: Visión desde las redes cubanas de investigación, Cuba, Editorial Feijóo.

${ }^{5}$ En el campo de estudios sociales la comprensión del enfoque sociológico es relevante pues en relación con otras ciencias que poseen el mismo objeto general [...] su intención [es la] de examinar la sociedad en su integralidad, como síntesis de la interacción de los más disímiles fenómenos particulares, y de jerarquizar, en condiciones históricas concretas, el conjunto de circunstancias y el tipo de sus nexos combinados que ejercen la influencia determinante en el comportamiento de diferentes procesos" (Espina, 1995: 39).
} 
incluyen los de la economía política, los estudios socioculturales y la integración disciplinar.

Se aplicó análisis de contenido bajo un muestreo no probabilístico de tipo intencional a 119 producciones científicas en función de los formatos de socialización y la representatividad de las disciplinas sociales que tienen como objeto problemáticas del espacio rural y agrario; los temas y la relación con las tradiciones teóricas y metodológicas, y con las políticas públicas; el reconocimiento social de los autores y la estabilidad de sus producciones durante el periodo; la representatividad de instituciones en la acumulación del conocimiento y de producciones que son referenciadas en otras, y por los agentes productores como relevantes por los aportes en el plano teórico y de contrastación empírica.

Las producciones abarcan desde 1980 hasta 2015, debido a que los años ochenta se reconocen como un momento superior en el desarrollo de la ciencia y de las ciencias sociales en particular. Crece la producción científica en esta etapa amparada por el carácter interdisciplinar del objeto, lo que acentúa la heterogeneidad y complejidad de los estudios. Se desarrollan programas de investigación y los procesos de formación en el tema agrario y rural; hay acumulación de un capital humano y por tanto, del capital social y cultural; mediante la formación se consolidan grupos y centros de estudios con líneas vinculadas directamente a la temática.

El cierre del periodo obedece a la etapa de reorganización del sistema de ciencia mediante el Decreto-Ley 32/2014. De las entidades de Ciencia, Tecnología e Innovación. El mismo establece criterios, como responder a necesidades del desarrollo de la economía y la sociedad; priorizar la ejecución de los encargos estatales; favorecer el mayor impacto de los resultados y actividades de inter-fase y de transferencia de tecnología. Además, coincide con el cierre de proyectos y la convocatoria a programas nacionales del ciclo 2016-2020.

$\mathrm{El}$ análisis incluye las determinaciones del conocimiento; éstas reflejan las intra-relaciones ${ }^{6}$ manifiestas en el ámbito universitario. Se recopilaron mediante entrevistas semiestructuradas a implicados en el proceso de producción de conocimientos (especialistas con 30 años o más de experiencia en la investigación, líderes de grupos científicos y formadores de profesionales) que describen los vínculos disciplinares e institucionales para la producción del conocimiento y los mecanismos para su legitimación.

Las implicaciones se establecen a partir de las contradicciones entre lo que establecen las normativas de cada uno de los procesos sustantivos de la universidad (formación, investigación), las relacionadas con las políticas públicas y el sector empresarial, y lo que aflora al contrastar mediante la práctica investigativa.

\section{Resultados}

En Cuba el uso social del conocimiento proviene de las particularidades heredadas desde las reformas planteadas por Francisco Frías y Jaccott, Enrique José Varona, Julio y Antonio Mella. ${ }^{7}$ Después de 1959 se profundiza la interrelación entre formación e investigación para el desarrollo en las universidades. Con la Reforma Universitaria en 1962 las universidades se convierten en centros multifacéticos, coexisten actividades docentes, de investigación y producción

\footnotetext{
${ }^{6}$ Por intra-relaciones se entienden las relaciones que se establecen al interior de las universidades cubanas, los modelos de formación de profesionales, el modo de producción de conocimiento y los indicadores que describen la gestión de la ciencia.

${ }^{7}$ Demuestran que la situación política era resultado de la estructura económica y su repercusión en la vida colectiva. Era necesaria una organización económica que cambiara el carácter monoproductor e importador de insumos, la nacionalización de latifundios y tierras improductivas, el establecimiento de un máximo de posesión y la prohibición para la adquisición de tierras a extranjeros. Asimismo los problemas sociales disminuirían con el cambio del modelo agrícola, su distribución espacial y de la fuerza laboral; también con la erradicación de carencias científicas y tecnológicas y del desconocimiento de la cultura e ideología nacional.
} 
(Consejo Superior de Universidades (CSU), 1962). Se crean institutos de investigación y se inicia una etapa de promoción de la ciencia; profesores y estudiantes universitarios se abocan a realizar investigaciones acerca de los requerimientos y las demandas sociales, económicas, culturales e históricas, y apoyan las prioridades del desarrollo económico y social del país, bajo el principio de inclusión y equidad social.

Con la tesis y resolución "Sobre Política Científica Nacional" (Partido Comunista de Cuba, 1978) se crean condiciones más favorables para el desarrollo de la actividad docente e investigativa en el campo de las ciencias sociales. Aunque es con la implementación de la Resolución 171 de 1987 de la Academia de Ciencias de Cuba (ACC) cuando la actividad científica se encamina hacia el perfeccionamiento y la integración con el sector académico y hacia la toma de decisiones. El nuevo Sistema de Ciencia e Innovación Tecnológica (SCIT) de 1996 coloca en su centro a la producción de bienes y servicios, sobre bases de eficiencia y competitividad para su inserción en el mercado internacional. ${ }^{8}$

De esta manera el modelo de universidad cubana refleja un carácter humanista, moderno y universalizado; científico, tecnológico e innovador e integrado a la sociedad, con su sector productivo, sus territorios y comunidades (Díaz-Canel, 2012). La Reforma Universitaria le otorga a la investigación científica un papel definitorio en el desarrollo universitario como un objetivo básico e irrenunciable. Éste permite un emparejamiento del desarrollo científico por todo el territorio nacional desde un modelo más apegado a las demandas del contexto.

La tercera misión viene a profundizar las estrategias implementadas por la educación superior. Los espacios rurales como objeto de investigación y mediante la docencia y la extensión universitarias en pos de la transformación social, dan origen a centros de estudio y grupos de investigación, principalmente en las universidades fuera de la capital del país. Éstos obtienen recursos para la investigación, la docencia y la intervención social, pese a la disciplinarización en la construcción del conocimiento y la ausencia de profesionalización del estudio acerca de lo rural en las ciencias sociales cubanas.

El ámbito rural, por sus potencialidades para el desarrollo, constituye un escenario de integración. El conocimiento científico producido integra saberes variados (gráfico 1) y en Cuba, de manera orgánica, vincula a profesionales de las ciencias sociales con los colectivos de los programas científico-técnicos como el de ciencias agropecuarias. Se desarrollan experiencias de extensión, el Sistema de Extensión Agraria se consolida como refiere Marzin et al. (2014), Cid (2017) y Salguero et al. (2018), aunque esta actividad no se institucionaliza en el país (Rodríguez et al., 2021). Esta problemática ejemplifica la mencionada contradicción de carácter científico que posibilita examinar las determinaciones e implicaciones sociales de la producción de conocimientos que toma a lo rural como contexto analítico en la relación entre desarrollo científico y educación superior.

Entre las determinantes sociales que actúan en el desarrollo científico y en particular el de las ciencias sociales está el doble circuito de producción y distribución del conocimiento; uno que se ocupa de los procesos de formación profesional y el otro de la investigación propiamente dicha. Las universidades, por un lado, y los centros de investigación del Ministerio de Ciencia, Tecnología y Medio Ambiente (CITMA) por otro, cuentan con características propias y con distintas formas de relacionarse con los procesos de toma de decisión, una conexión mucho más orgánica en los segundos.

\footnotetext{
${ }^{8} \mathrm{El}$ nuevo sistema de ciencia se ajusta al modelo restringido de sistemas de innovación, donde el sector de I+D se coloca en el centro de la política, aunque despliega mecanismos para fomentar las actividades de I+D, los vínculos universidad-empresa y la creación de empresas de base tecnológica (Núñez y Montalvo, 2015).
} 


\section{Gráfico 1. Distribución disciplinar de las producciones científicas}

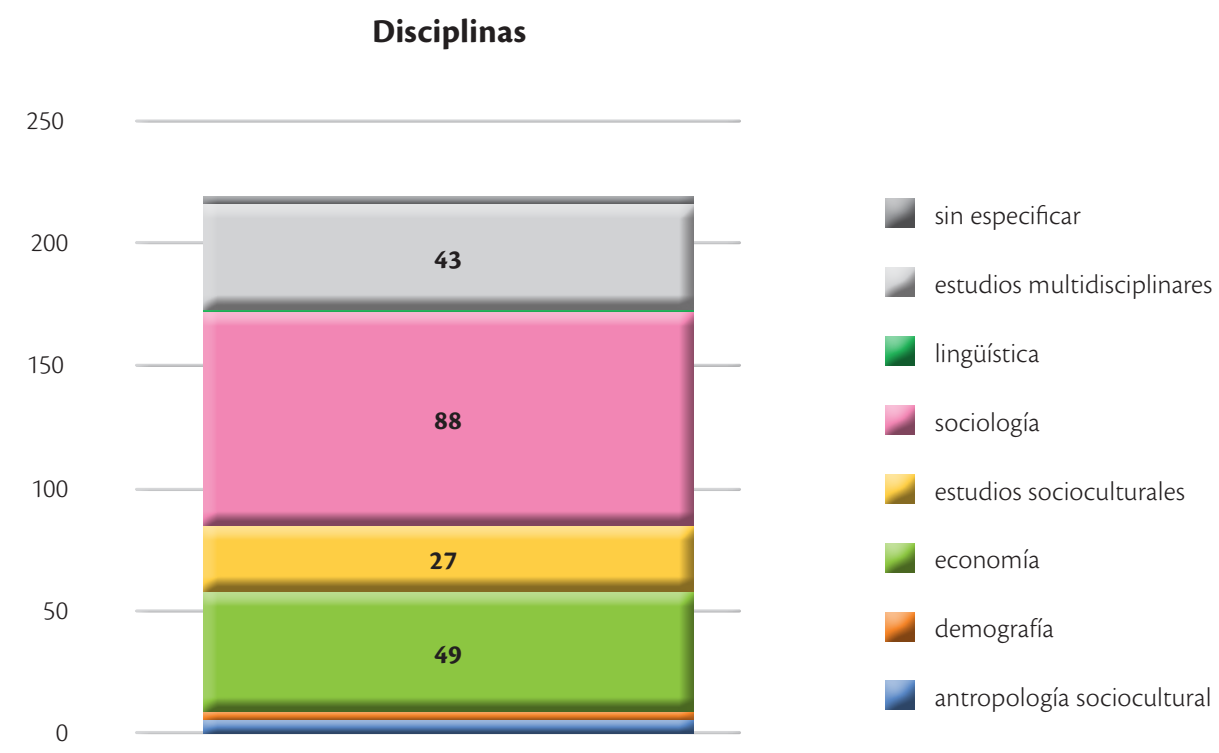

Fuente: elaboración propia (2019) a partir de base de datos de producciones científicas rurales (Herrera, 2019).

El Sistema de Ciencia e Innovación Tecnológica (SCIT) reconoce a ambos en su estructura junto a los órganos encargados de la dirección, planificación y organización; así como los dispositivos legales que ordenan la actividad de ciencia, tecnología e innovación. Pero sólo mediante el cumplimiento de la tercera misión de las universidades es posible constatar el reconocimiento social. La pertinencia del desarrollo científico universitario debe enfrentar implicaciones en procesos de legitimación de la ciencia enfocados al desarrollo nacional.

Se preconcibe la legitimación del conocimiento por la diferenciación de los campos de investigación, la adscripción disciplinar e institucional. La utilidad del conocimiento producido está en el conjunto de contribuciones teóricas, metodológicas y empíricas obtenidas por las universidades como una totalidad. La fragmentación de los saberes unido a procesos discontinuos en la formación, determinan las interacciones del SCIT. La priorización de objetos de investigación en las ciencias sociales en función de condicionantes económicas conlleva al apoyo financiero estatal de unas líneas, el detrimento o la escasez de análisis sobre temas fundamentales en la agenda como la movilidad y envejecimiento de la fuerza de trabajo; las estrategias de reproducción social de clases, grupos y capas, la pobreza, la violencia, estudios sobre familias.

Estas generalidades permiten contextualizar el análisis que las ciencias sociales cubanas aportan al conocimiento sobre lo rural. De igual manera se corroboran indicadores de productividad, legitimidad y difusión del conocimiento (tabla 1) reveladores de las determinaciones del conocimiento, las relaciones en el ámbito universitario y las implicaciones sociales en el uso del mismo. 
Tabla 1. Indicadores de productividad, legitimidad y difusión del conocimiento

\begin{tabular}{|c|c|c|}
\hline Dimensión & Modo 1 & Modo 2 \\
\hline \multirow{2}{*}{ Productividad } & $\begin{array}{l}\text { Tipos de producciones: trabajos de cursos, tesis } \\
\text { de diplomas, artículos con fines docentes. }\end{array}$ & $\begin{array}{l}\text { Publicaciones científicas indizadas en revistas main stream, bases de datos } \\
\text { de prestigio internacional (BDI) y web de la ciencia (WC); patentes, asesorías, } \\
\text { transferencia de tecnología mediante la exportación. }\end{array}$ \\
\hline & $\begin{array}{l}\text { Grados académicos otorgados (formación de } \\
\text { masters y doctores); becas de investigación. }\end{array}$ & $\begin{array}{l}\text { Premios nacionales de investigación e innovación tecnológica otorgados por } \\
\text { la Academia de Ciencias de Cuba (ACC); solución de problemas prácticos; } \\
\text { transmisión de educación científica a la población. }\end{array}$ \\
\hline Legitimidad & $\begin{array}{l}\text { La validación se hace por la comunidad de } \\
\text { especialistas; tiene una fundamentación } \\
\text { disciplinar. }\end{array}$ & $\begin{array}{l}\text { La validación se asocia a la negociación de actores diversos (sector productivo, } \\
\text { mercado, gobierno, sociedad en general) frente a las demandas; tiene una } \\
\text { fundamentación inter y transdisciplinar. }\end{array}$ \\
\hline Difusión & Se concentra en el ámbito institucional. & $\begin{array}{l}\text { Percepción pública y participación ciudadana: posibilita la relación } \\
\text { conocimiento científico-sociedad. }\end{array}$ \\
\hline
\end{tabular}

Fuente: elaborado por Herrera (2020), basado en Vessuri (1987); Núñez (2008) y Roa-Mendoza (2016).

A partir de las producciones científicas acumuladas desde la economía, la sociología, antropología social, demografía y su integración, se constatan las determinaciones sociales del conocimiento. En primera instancia se señala que de las producciones científicas identificadas sobre lo rural, el 79\% son producidas por las universidades y los centros de investigación adscritos a éstas (gráfico 2).

A partir de los indicadores de productividad, legitimidad y difusión del conocimiento presentados en la tabla 1 y los formatos de socialización de los resultados científicos, coexisten tipos de producciones tanto del modo 1 como del modo 2. Se constata la implementación de la investigación en los procesos formativos de grado y pregrado; sobre todo el modo 2 conduce a resultados de investigación que se encaminan a ampliar la solución de demandas sociales y garantizar una legitimidad, validada no sólo por la comunidad de especialistas (gráfico 3).

La indagación confirma que muchas producciones son el resultado de proyectos de investigación en los que las universidades cooperan con organismos internacionales (FAO, PNUD, Agencia Suiza para la Cooperación (COSUDE), Agencia Canadiense para el Desarrollo Internacional, CIDA); organismos gubernamentales (CITMA, el Órgano de Montañas, el Ministerio de la Agricultura); organismos no gubernamentales (Asociación Nacional de Agricultores Pequeños (ANAP), la Asociación Cubana de Producción Animal (ACPA), la Asociación Cubana de Técnicos Agrícolas y Forestales (ACTAF), organizaciones de masas como la Federación de Mujeres Cubanas, y formas productivas agropecuarias.

Se corroboran así las interrelaciones de los componentes externos: universidades, el sector productivo y estructuras gubernamentales. Los estudios de corte institucional o sobre las políticas agrarias, la interrelación con los ejes de desarrollo, posicionan al sector agrario como potencialidad para el desarrollo de los municipios en Cuba. El enfoque del desarrollo territorial rural, a partir del primer lustro del siglo XXI, emerge como una realidad entre la innovación social y el entorno; los actores sociales, el territorio y el ambiente son articulados para enfrentar la heterogeneidad del mundo rural y trazar planes de desarrollo agrícola y rural sostenibles (Herrera, 2020). 
Gráfico 2. Distribución de las producciones científicas por adscripción SCIT

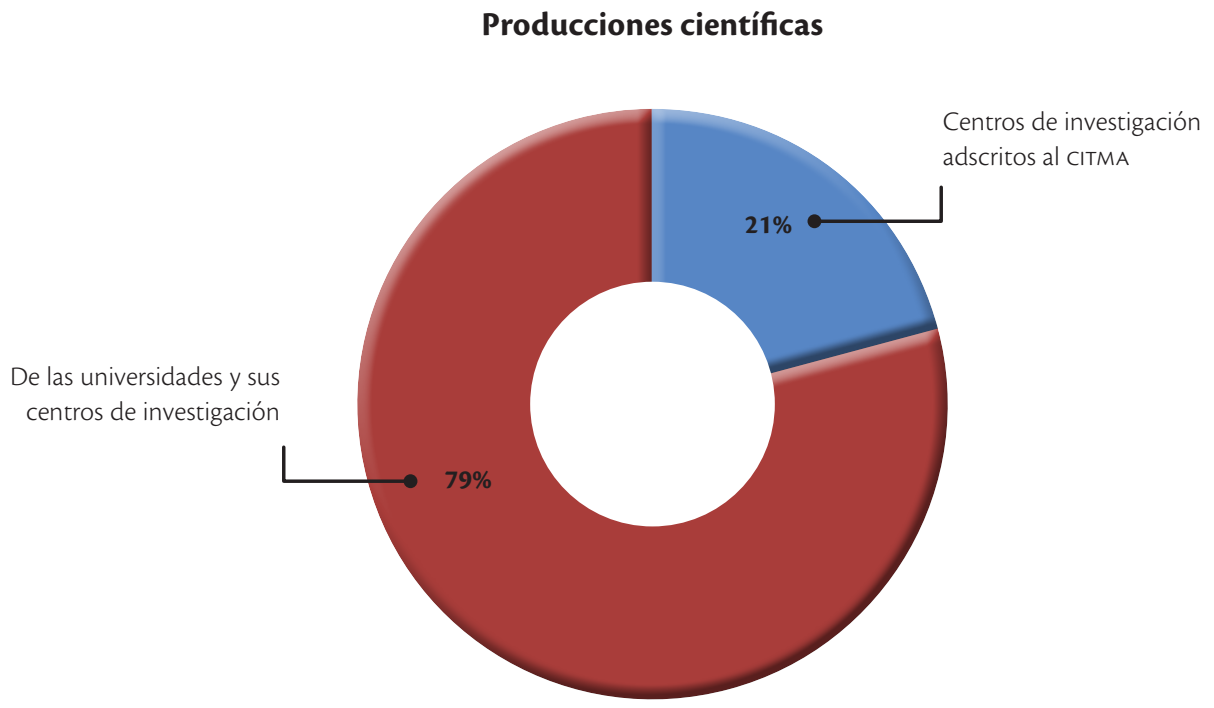

Fuente: elaboración propia (2019) a partir de base de datos de producciones científicas rurales (Herrera, 2019).

Gráfico 3. Formato de socialización de los resultados de la investigación científica Formato de socialización

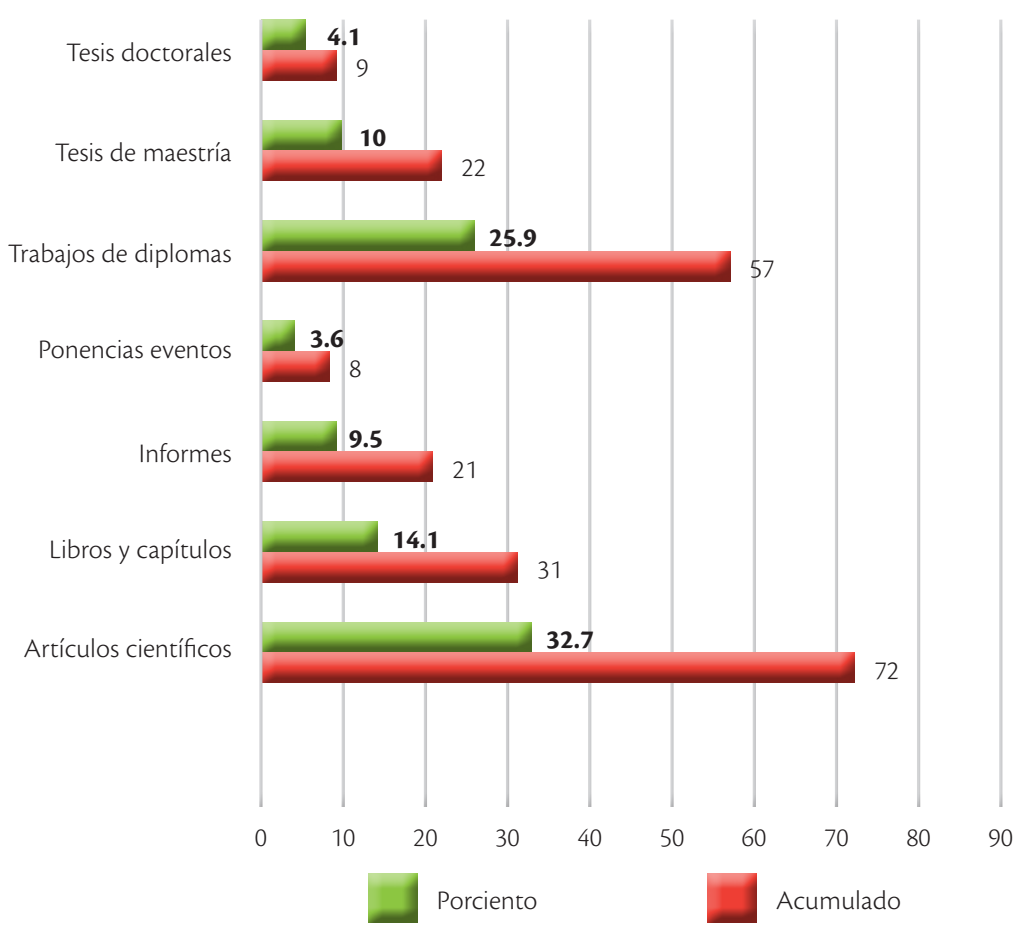


Sin embargo, la intensidad de la relación de la universidad con el sector productivo y las estructuras gubernamentales no se traduce en el reconocimiento de la legitimidad del conocimiento al formular políticas públicas. Existen pocas producciones que describan procesos de transformación social. Además, los actores del proceso de producción de conocimiento coinciden en valorar que ha predominado la incomunicación y la falta de diálogo entre la academia y los decisores. Aquí radica uno de los obstáculos que encuentran los estudios rurales y su contribución al desarrollo agropecuario en el país.

Sobre esta determinación influyen otras características de la investigación desarrollada por las universidades y en particular desde las ciencias sociales: marcado carácter descriptivo; una modificación de estudios cuantitativos y perspectivas macro-sociales a estudios que potencian la perspectiva cualitativa con un enfoque micro-social.

La disciplinarización de la investigación ha influido en el reconocimiento de las investigaciones. En la legitimación del conocimiento producido han primado los estudios económicos, pero los investigadores reconocen que, al no visibilizar quiénes son los que están detrás de esa economía y los procesos de interrelación social, se han limitado algunas de las decisiones que en políticas se han tomado. Las interacciones son influidas por tradiciones teóricas, hegemónicas, que conllevan al desarrollo de contradicciones en la ciencia, originadas en las etapas de formación académica. De igual manera contribuyen a potenciar temáticas de conocimiento que se acumulan, establecen agendas de investigación y permiten la creación de centros de investigación y grupos de estudio que legitiman la preponderancia y centralidad de problemáticas sociales de los escenarios rurales.

A pesar de esta tendencia, la complejidad de las problemáticas sociales, el nivel de integración de investigadores y académicos propicia la combinación de saberes desde la inter y transdisciplinariedad.
Pero, particularidades del proceso formativo de pregrado (Herrera, 2017) intervienen en la validación del conocimiento, la disciplina científica propicia la segmentación del saber y es fuente para su legitimación. Se adiciona a las relaciones disciplinares, la inserción en las redes de socialización de la ciencia. El criterio de publicación en revistas de mainstream, BDI o WC genera más prestigio y reconocimiento social que la generalización de los resultados en la práctica social o la formulación de políticas públicas.

Estos resultados conllevan a analizar las implicaciones de las relaciones entre los actores fundamentales para el desarrollo en los escenarios rurales, que no sólo incluye a quienes producen y acumulan conocimientos científicos, sino a otras instituciones gubernamentales y estructuras territoriales: formas productivas del sector agropecuario, comunidades, organismos de la administración central del Estado.

Este complejo entramado de articulaciones que impactan en la sociedad rural impone a las ciencias sociales una capacidad crítica para reflexionar acerca de las contribuciones de cada una y los discursos como generadores de las determinaciones. Pero estos discursos, necesariamente, sólo pueden ser comprendidos según el contexto en que se producen. En el modelo de desarrollo cubano el proceso de crecimiento económico está aparejado a la equidad y justicia social; hay una atención simultánea a los problemas económicos y sociales, en ocasiones se ha dado prevalencia a lo social en detrimento de lo económico. En diferentes etapas se ha potenciado uno o más sectores de la economía como motores del desarrollo; pero en el análisis de la problemática rural hay un interés casi permanente por el desarrollo agrario.

El contexto nacional apuesta por el desarrollo científico como impulsor del desarrollo económico y social; por tanto, el conocimiento científico constituye una meta, una demanda. Ya se ha expuesto que la validación de los discursos depende de esquemas disciplinares e institucionales, heredados de 
un academicismo en las ciencias sociales cubanas. El desarrollo de éstas es diverso y desigual; su organización presenta cierto retraso con respecto a las ciencias naturales y técnicas. Se advierten elementos de fragmentación de los saberes; la red de información que permite el flujo de intercambio de resultados obtenidos entre los centros es insuficiente; existen barreras institucionales y proyectos de investigación que limitan los enfoques problemáticos inter y multidisciplinarios (Espina, 1995; Hernández et al., 1997; Limia, 1995; Guanche et al. 2012).

También se percibe una desconexión entre las políticas públicas agrarias y las necesidades de desarrollo de las comunidades rurales donde se asientan los usufructuarios y productores agropecuarios en general (Leyva y Arias, 2015; Leyva, 2016). Influye en esta desconexión la proporcionalidad de la formación posgraduada con respecto a la investigación de las problemáticas sociales sobre lo rural: 24 tesis de maestría (8.6\%), 9 tesis de doctorado $(3.2 \%)$ y 57 trabajos de diploma (20.5\%). La transferencia de conocimiento incorporado en graduados de doctorado implica una profundización en el nivel científico de la investigación y asegura la calidad de la educación superior (Saborido, 2018), pero aún no llega a alcanzar, en cifras ni en resultados, el alto nivel esperado con respecto a la producción, de manera que contribuya a la satisfacción de necesidades sociales y culturales.

El diseño de las instituciones de investigación de las ciencias sociales tiende a ser disciplinario, pero las exigencias en las investigaciones tienden a la transdisciplinariedad. Los productores de conocimiento plantean que cambiar la estructura de las instituciones de disciplinaria a problémica sería complejo, como lo es cambiar la mentalidad de los investigadores para un trabajo transdisciplinar. Sin embargo, se identifica una labor cooperada, aún insuficiente, entre las instituciones y entre los investigadores.

El partidismo de la ciencia (Rodríguez, 1983; Rojas, 1983 $)^{9}$ conduce a prácticas no esperadas: el uso ideologizante de la teoría; los resultados científicos con mucha frecuencia confirman y/o fundamentan los impactos positivos de políticas implementadas, pero no siempre reflejan de manera crítica las contradicciones entre los resultados esperados y los impactos sobre las dinámicas sociales reales.

Las operacionalizaciones ofrecidas en la investigación científica sobre lo rural no coinciden con los indicadores utilizados por las instituciones y organismos gubernamentales para delimitar lo rural. ${ }^{10}$ Las contribuciones empíricas desbordan el conocimiento priorizado y resaltan la necesidad de legitimar la concepción de que los cambios en la relación con la tierra son esenciales, pero los producidos a nivel subjetivo y en el orden super-estructural, que se manifiestan paulatinamente y de forma heterogénea, deben concretar más la comprensión e incorporación de la investigación como una necesidad de la práctica social y el establecimiento de políticas.

\section{Discusión}

El desarrollo alcanzado por las ciencias sociales y el reconocimiento al conocimiento producido por las mismas marca de manera particular las interrelaciones que se establecen entre los actores del desarrollo.

\footnotetext{
${ }^{9}$ El principio del partidismo consciente en las ciencias sociales, como condición y no como obstáculo, regula el análisis objetivo de los juicios valorativos. Debe servir de apoyo y base orientadora al criterio oficial u ofrecer sustentación teórica o para aportar juicios, argumentos y conclusiones que puedan contribuir a modificaciones o rectificaciones necesarias (Rodríguez, 1983). La Política Científica de las Ciencias Sociales y Humanísticas enuncia: "No hay ciencias sociales neutrales, el estudio de sociedad es siempre desde la sociedad y no al margen ni por encima de ella" (CITMA, 2002).

${ }^{10}$ Desde el Censo de Población y Viviendas de 1981 existe una posición ideológicamente hegemónica que garantiza la estructuración de políticas públicas, la definición de rural se estipula por límites poblacionales y se complementa con indicadores funcionales: "Población residente en lugares habitados por menos de 500 personas o la de aquellos con más de 500 y menos de 2 000, que presentaron menos de cuatro de las características urbanas: alumbrado público, calles pavimentadas, acueducto, red de alcantarillado, cloacas, servicios médico asistencial y centro educacional".
} 
De acuerdo con los diferentes presupuestos teóricos planteados, en la experiencia de los estudios sociales rurales se mantiene un nivel lineal en el papel de la universidad, al ofrecer estrategias para regular el funcionamiento del gobierno y su relación con otros agentes públicos.

Las investigaciones reflejan un ideal en la participación del gobierno durante el proceso de producción de conocimiento; pero en gran número de éstas, sólo es una participación basada en el suministro de información o consultiva. Se hace imprescindible una participación funcional e interactiva del gobierno de manera que garantice el monitoreo y el ajuste en los procesos de transformación social y en la implementación y evaluación de los programas, proyectos y planes de desarrollo.

Las interrelaciones entre los actores y sus resultados sobre el desarrollo, con un basamento científico, presupone una mirada multidimensional a las ciencias sociales (geográfico, económico, político, social, cultural) que limite el privilegiado análisis disciplinario. Para el caso de los estudios sociales rurales no es posible un análisis tradicional de la ciencia, la naturaleza de las problemáticas sociales exige de la convergencia de varias disciplinas científicas (Herrera y Muñoz, 2017).

No se puede hablar de un Sistema Nacional de Innovación, las políticas científicas aún no logran actualizarse en comparación con las experiencias prácticas que están produciendo conocimiento e innovación, desde la cooperación entre agentes. Aunque la investigación social sobre lo rural tiene un origen en instituciones que tradicionalmente se adscriben al modo 1 de producción de conocimiento, la difusión del mismo trasciende los límites institucionales. Se carece de espacios para la retroalimentación con la esfera pública.

Sobre todo, los esquemas de interacción entre actores o esferas institucionales representados por el gobierno, las empresas y las universidades, están mediados por las implicaciones que supone en primer lugar, transformar las intra-relaciones. Queda mucho por transformar en los programas de formación de profesionales, principalmente, para investigar e intervenir en problemáticas como las rurales, que requieren de un conocimiento científico que interseccione el estudio de las relaciones sociales, la producción agroindustrial y la implementación de políticas públicas.

La problemática rural y agraria en Cuba tiene una tradición en la investigación, pero los análisis científicos muestran que las políticas sectoriales, después de 1959, tienen una conexión entre el protagonismo estatal para el desarrollo agrícola y rural con una prioridad macroeconómica y social. Sin embargo, al interior de las vinculaciones de los actores que conforman la Triple Hélice, existen carencias en las alternativas de focalización necesarias en los espacios rurales. Predomina el enfoque sectorial sobre el territorial-integral.

Las ciencias sociales producen conocimientos que difieren de su utilidad para lo académico y para el propósito analítico, que contribuyen a generar cambios institucionales. Lo rural y lo agrario, en el centro de los debates académicos y científicos, provocan incongruencias en la toma de decisiones. Aunque estas diferencias se entienden como una continuidad en el estudio de la producción agrícola, la agroindustria, el mercado y el consumo, la ampliación o restricción del objeto de estudio inducen a distorsiones para la operacionalización. El límite poblacional, complementado con criterios funcionales, estipulado para Cuba (nota 10), no es suficiente para la toma de decisiones por parte del sector productivo. Para la sociedad en general también resulta limitante, pues los asentamientos poblacionales no son homogéneos, existen múltiples ruralidades.

Las determinaciones del conocimiento y las implicaciones sociales, como objeto de análisis, evidencian que en la relación entre el conocimiento científico producido por las universidades y el desarrollo, los elementos teóricos en su contrastación 
empírica presentan las particularidades del contexto. El cumplimiento de la tercera misión de las universidades queda trunco sin la valoración de éstas: escasa satisfacción en la dimensión de servicio público, en la satisfacción de demandas sociales, económicas, culturales; una formación de profesionales que no logra mantener los niveles de interés frente a las problemáticas rurales al comparar el pregrado con el posgrado. El nivel de contribución entre los integrantes, apunta hacia proyectos de cooperación y no hacia proyectos de investigación-desarrollo que conlleven al fomento del emprendedurismo y al establecimiento de políticas públicas.

Para la investigación social, la generación, implementación o evaluación de políticas públicas constituyen el mecanismo regulador para las acciones de los otros actores de la Triple Hélice; los diagnósticos y datos acerca del proceso de transformación socioeconómica ponen al gobierno como referente para la toma de decisiones. De esta manera, las implicaciones acerca de la delimitación de lo rural determinan el curso de las concepciones del desarrollo como proceso general.

El SCIT sufre antes las limitaciones que presentan las ciencias sociales para legitimar el conocimiento sobre lo rural. Aun cuando presentan un alto contenido científico y en muchos casos contienen recomendaciones a las políticas públicas, su objeto, la operacionalización de las variables, y su propósito, no es el de formular instrumentos para fortalecer el sistema de instituciones y de la política en general: se definen en un rol técnico, ofrecen datos, en vez de ser consultores efectivos del ciclo de las políticas públicas. Las contribuciones manifiestas en la investigación desarrollada por las universidades, por su naturaleza, se restringen por el rol de gobierno que exige una mayor integración de los actores sociales para el desarrollo rural.

No obstante, un cambio en la perspectiva analítica para las ciencias sociales sitúa la reflexividad como requisito que potencia el análisis permanente en torno a qué produce la ciencia acerca de los espacios rurales, cómo lo produce y qué utilidad tiene. La pertinencia de la investigación, la docencia y la extensión para la transformación social se amplía una vez que las concepciones que desarrollan los diversos actores pueda pensar la ruralidad más allá de un espacio físico, de realización de prácticas económico-productivas y se genere una concepción dinámica, incluyente, transdisciplinar de la investigación universitaria.

\section{Consideraciones finales}

La tradición del desarrollo científico ha producido una manera particular de examinar los procesos económico-productivos, políticos, sociales, culturales. Aunque en los últimos años se ha generado una tendencia hacia la articulación, los modelos teóricos representan una separación entre ramas del saber para la producción y legitimación del conocimiento. En el análisis de las problemáticas rurales no pueden ser excluidos los procesos y relaciones sociales, pues el ser humano es quien produce y establece las relaciones de reproducción de la vida. En este sentido, son las ciencias sociales las que permiten discernir el papel de los componentes de los sistemas de ciencia e innovación, los actores y redes, sus interrelaciones e intra-relaciones en los modelos, enfoques y teorías relacionados con el análisis del conocimiento producido, legitimado y difundido.

Para los estudios sociales rurales en Cuba, las universidades alcanzan su pertinencia social, garantizan la reproducción del conocimiento y la transformación social mediante la investigación. El conocimiento producido es la base para un desarrollo rural que todavía debe afrontar un rol más activo de la educación superior en la producción científica; incongruencias entre políticas públicas que potencien las alternativas de focalización y su correspondencia con el protagonismo estatal-universalidad-prioridad macroeconómica; y un equilibrio entre lo económico y lo social. El análisis de las implicaciones y 
determinaciones sociales de la producción de conocimientos es sólo un punto de origen para desarrollar las ciencias en función de las necesidades de la sociedad y el rol de la educación superior.

Las ciencias sociales, mediante la investigación en el contexto cubano, ofrecen conocimientos científicos

\section{Referencias}

Batista, Mario y Julio Pérez (2016), "Modelo y metodología para la gestión de la ciencia y la innovación en las universidades", Revista Cubana de Educación Superior, vol. 35, núm. 2, mayo-agosto, pp. 154-168.

Casas, Rosalba (2015), "La literatura sobre la vinculación: procesos interactivos entre actores, redes de conocimiento, espacios regionales", Taller: Análisis de experiencias devinculación academia-sector productive (9-12 de febrero), La Habana.

Castro, Fidel (1965), Discurso pronunciado por el Comandante Fidel Castro Ruz, Primer Secretario del PURSCy Primer Ministro del gobierno revolucionario, en la concentración conmemorativa del sexto aniversario de la Revolución (2 de enero), La Habana, Departamento de versiones taquigráficas, en <http://www.cuba.cu/gobierno/discursos/1965/ esp/f020165e.html> [Consulta: febrero de 2018].

CEPAL (1990), Transformación productiva con equidad. La tarea prioritaria del desarrollo de América Latina y el Caribe en los años 90, Santiago de Chile, <https://www.cepal.org/ es/publicaciones/2102-transformacion-productivaequidad-la-tarea-prioritaria-desarrollo-america-latina> [Consulta: junio de 2018].

CEPAL-UNESCO (1992), Educación y conocimiento: eje de la transformación productiva con equidad, Santiago de Chile, <https://repositorio.cepal.org/handle/11362/2130> [Consulta: junio de 2018].

Cid, Greco (2017), "Investigación agronómica y extensión agraria en Cuba: unidad dialéctica imprescindible para de utilidad para el desarrollo rural. El cumplimiento de las misiones universitarias, principalmente la tercera, puede lograr una transformación de los contextos integrando a todos los actores, adecuando demandas y potencialidades desde la relación universidad-ciencia-desarrollo.

lograr seguridad alimentaria", Revista Ingeniería Agrícola, vol. 3, núm. 3, pp. 35-38.

Consejo Superior de Universidades (CSU) (1962), La reforma de la enseñanza superior en Cuba, La Habana, Universidad de La Habana (Colección Documentos).

Coraggio, José Luis (2002), "Universidad y desarrollo local”, en Seminario Internacional La educación superior y las nuevas tendencias, Quito, Consejo Nacional de Educación Superior (CONESUP)/UNESCO/CIESPAL.

Díaz-Canel, Miguel (2012), "Hacia un mayor impacto económico y social de la educación superior", Revista Universidady Empresa, vol. 8, núm. 1, pp. 3-10.

Engel, Paul (2001), Facilitando el desarrollo sostenible: ¿hacia una extensión moderna?, Santiago de Chile, CEDRO.

Entrena, Francisco (1998), Cambios en la construcción social de lo rural. De la autarquía a la globalización, Madrid, Tecnos.

Espina, Mayra (1995), "Tropiezos y oportunidades de la sociología cubana", Revista Temas, núm. 1, enero-marzo, pp. 36-49.

Etzkowitz, Henry (2004), "The evolution of entrepeneurial university", International fournal of Technology and Globalisation, vol. 1, núm. 1, pp. 64-77.

Etzkowitz, Henry y Loet Leydesdorff (2000), "The dynamics of innovation from national systems and "mode 2" to a Triple Helix of university-industry government relations", Research Policy, núm. 29, pp. 109-123. 
Etzkowitz, Henry y Loet Leydesdorff (1997), University and the global knowledge economy. A Triple Helix of universityindustry-government relations, Londres, Pinter Publishers.

Gaceta Oficial de la República de Cuba (2014), Decreto-Ley No. 323 De las entidades de ciencia, tecnología e innovación, núm. 37 (29 de agosto), La Habana, Consejo de Estado.

Gibbons, Michael et al. (1997), La nueva producción de conocimiento. La dinámica de la ciencia y la investigación en las sociedades contemporáneas, Barcelona, Ediciones PomaresCorredor.

González, Gil y Mercedes González (2006), “Extensión universitaria: una aproximación conceptual desde la perspectiva cubana", Revista Cubana de Educación Superior, vol. 26, núm. 2, pp. 69-76.

González, Gil y Mercedes González (2003), "Extensión universitaria: principales tendencias en su evolución y desarrollo", Revista Cubana de Educación Superior, vol. 23, núm. 1, pp. 15-26.

Guanche, Jesús et al. (2012), Informe preliminar sobre el estado de las ciencias sociales y humanísticas de cara al cumplimiento de los Lineamientos de la politica económica y social del Partido y la Revolución y de los objetivos de trabajo aprobados en la Primera Conferencia Nacional, La Habana, <http://www. academiaciencias.cu/sites/default/files/adjuntonoticias/ ANEXO\%202.\%20CSH.\%20Informe..pdf> [Consulta: abril de 2019].

Hernández, Rafael et al. (1997), "Las ciencias sociales en la cultura cubana contemporánea", Revista Temas, núm. 9, enero-marzo, pp. 68-86.

Herrera, Yisel (2020), "Estudios sociales rurales: campo y producciones científicas", Revista Mexicana de Sociología, vol. 82, núm. 2 (abril-junio), pp. 281-309.

Herrera, Yisel (2019), "Los estudios sociales rurales en Cuba. Un campo constituido", Tesis en opción al grado del Doctor en Ciencias Sociológicas, La Habana, Departamento de Sociología, Universidad de La Habana.

Herrera, Yisel (2017), "Aspectos sociales para la formación del profesional agrícola en Cuba. Apuntes para un debate", Revista Científica Agroecosistemas, vol. 5, núm. 2, pp. 106-114.
Herrera, Yisel y Teresa Muñoz (2017), "El desarrollo científico y académico de lo rural en las ciencias sociales en Cuba. Aproximaciones", Redes. Revista de estudios sociales de la ciencia y la tecnología, vol. 23, núm. 45, diciembre, pp. 139-161.

Leyva, Arisbel (2016), "Políticas públicas campesinas en Cuba: la equidad y otros desafíos", Revista Temas, núm. 83, julio- septiembre, pp. 12-19.

Leyva, Arisbel y María Arias (2015), "Cuba: reproducción y estructura social del campesinado. Desafios para las políticas agrarias y rurales", en Congreso de la Asociación Latinoamericana de Sociología, <http://sociologia-alas.org/ acta/2015/GT-05/Cuba\%20reproducci\%C3\%B3n\%20 y $\% 20$ estructura $\% 20$ social $\% 20 \operatorname{del}^{\circ} \% 20$ campesinado $\% 20$ d e s a f $\%$ C $3 \%$ A D o s $\% 20$ pa r a $\% 201$ a s $\% 20$ pol\%C.3\%ADticas\%20agrarias\%20y\%20rurales.docx> [Consulta: junio de 2019].

Limia, Miguel (1995), “¿Hacia dónde van los estudios sociales?", Revista Temas, núm. 1, enero-marzo, pp. 18-26.

Martín, Juan Luis (1999), "La investigación social en Cuba (1959-97)", Revista Temas, núms. 16-17, octubre 1998junio 1999, pp. 143-153.

Marzin, Jacques et al. (2014), Herramientas metodológicas para una extensión agraria generalista, sistémica y participativa. Proyecto de apoyo al Sistema de Extensión Agraria en Cuba, La Habana, Editora Agroecológica, < http://agris.fao.org/ agris-search/search.do? recordID=FR2017103635> [Consulta: junio de 2021].

Ministerio de Ciencia, Tecnología y Medio Ambiente (CITMA) (2002), Política Científica para las Ciencias Socialesy Humanísticas. Resolución No. 132/2002, 19 de noviembre, Guba, CITMA.

Núñez, Jorge (2008), "Indicadores y relevancia social del conocimiento", en Irene Trellez y Miriam Rodríguez, Universalización y cultura científica para el desarrollo local, La Habana, Félix Varela, pp. 77-102.

Núñez, Jorge (1999), La ciencia y la tecnología como procesos sociales. Lo que la educación científica no debería olvidar, La Habana, Félix Varela.

Núñez, Jorge y Luis Montalvo (2015), "La política de ciencia, tecnología e innovación en Cuba y el papel de 
las universidades", Revista Cubana de Educación Superior, 34(1), pp. 29-43.

Organización de las Naciones Unidas para la Educación, la Ciencia y la Cultura (UNESCO) (2005), Informe Mundial de la UNESCO. Hacia las sociedades del conocimiento, París, UNESCO.

Organización de las Naciones Unidas para la Educación, la Ciencia y la Cultura (UNESCO) (1998), Conferencia mundial sobre la educación superior. La educación superior en el siglo XXI: Visión y Acción, Paris, UNESCO.

Partido Comunista de Cuba (1978), "Sobre la Plataforma Programática de Partido", en I Congreso del PCC. Tesis y Resoluciones, La Habana, Editorial Ciencias Sociales, pp. 3-126.

Pavón, María (2014), "Extensionismo en Cuba: estudios de caso", Cultivos Tropicales, vol. 35, núm. 1, pp. 5-10.

Roa-Mendoza, Claudia (2016), "Investigación en modo 3: una alternativa para la articulación investigación e intervención en educación superior", Revista Científica Guillermo de Ockham, vol. 14, núm. 2, pp. 103-110.

Rodríguez, Zaira (1983), "El partidismo objetivo como principio rector de las investigaciones sociales en la Cuba revolucionaria", Revista Cubana de Ciencias Sociales, núm. 1, pp. 38-48.

Rodríguez, Niurlys et al. (2021), "Formación de postgrado en Extensión Agraria: experiencias en Holguín, Cuba", Revista Iberoamericana de Educación Superior (RIES), vol. XII, núm. 33, DOI: https://doi.org/10.22201/ iisue.20072872e.2021.33.863. [Consulta: junio de 2021].

Rojas, Iliana (1983), "Algunas consideraciones acerca del principio del partidismo y el análisis de la estructura socio-clasista en La Historia me Absolverá", Revista Cubana de Ciencias Sociales, núm. 2, pp. 25-37.

Sábato, Jorge y Natalio Botana (1975), La ciencia y la tecnología en el desarrollo futuro de América Latina, <http:// docs.politicascti.net/documents/Teoricos/Sabato_ Botana.pdf> [Consulta: abril de 2020].

Saborido, José Ramón (2018), "Universidad, investigación, innovación y formación doctoral para el desarrollo en Cuba", Revista Cubana de Educación Superior, núm. 1, pp. 4-18.

Salguero, Zulema et al. (2018), "La extensión agraria en la educación superior en Cuba", Pedagogía Universitaria, vol. 23, núm. 1, pp. 16-28.

Schoen, Antoine et al. (2006), Strategic management of university research activities, Methodological guide, PRIME Project, Observatory of the European University, <http://www.enid-europe.org o www.prime-now. orgou> [Consulta: abril de 2020].

Serna, Gonzalo (2007), "Misión social y modelos de extensión universitaria: del entusiasmo al desdén", Revista Iberoamericana de Educación, núm. 43, pp. 3-25.

Souza, José et al. (2001), La cuestión institucional: de la vulnerabilidad a la sostenibilidad institucional en el contexto del cambio de época, San José, Proyecto ISNAR "Nuevo paradigma" (Serie Innovación para la Sostenibilidad Institucional).

Vessuri, Hebe (1987), "Los papeles culturales de la ciencia en los países subdesarrollados", en J. Saldaña, El perfil de la ciencia en América, México, Cuadernos de Kipu 1.

\section{Cómo citar este artículo:}

Herrera-Martínez, Yisel y Alicia Martínez-León (2022), “Experiencias de la relación universidad-ciencia-desarrollo en los estudios sociales rurales en Cuba”, Revista Iberoamericana de Educación Superior (RIES), vol. XIII, núm. 36, pp. 63-82, DoI: https://doi.org/10.22201/ iisue.20072872e.2022.36.1184 [Consulta: fecha de última consulta]. 